Research Article

\title{
Synchronization of Time Delay Coupled Neural Networks Based on Impulsive Control
}

\author{
Jie Fang $\mathbb{D},{ }^{1,2}$ Yin Zhang, ${ }^{1}$ Danying $X u^{1}$, and Junwei Sun $\mathbb{D}^{1,2}$ \\ ${ }^{1}$ College of Electric and Message Engineering, Zhengzhou University of Light Industry, Zhengzhou 450002, China \\ ${ }^{2}$ Henan Key Lab of Information-Based Electrical Appliances, Zhengzhou 450002, China \\ Correspondence should be addressed to Junwei Sun; junweisun@yeah.net
}

Received 25 June 2020; Accepted 30 July 2020; Published 18 August 2020

Guest Editor: Hou-Sheng Su

Copyright (@) 2020 Jie Fang et al. This is an open access article distributed under the Creative Commons Attribution License, which permits unrestricted use, distribution, and reproduction in any medium, provided the original work is properly cited.

This paper investigates the impulsive synchronization of time delay coupled neural networks. Based on the Lyapunov stability theory and impulsive control method, a distributed delayed impulsive controller is designed to realize synchronization of the coupled neural networks. A new impulsive delayed inequality is proposed, where the control effect of distributed delayed impulses is fully considered. In addition, a suitable Lyapunov-like function is established to prove the stability of the synchronization system. Numerical simulation examples are introduced to illustrate the effectiveness and feasibility of the main results.

\section{Introduction}

Neural networks are a type of mathematical model that simulates the thinking patterns of the human brain, which is used to imitate the structure and thinking mode of neural network in the human brain [1-6]. The neural network learning is to abstract and simplify the human brain from the microscopic structure and function. It is one of the ways to realize artificial intelligence. Since 1980s, the research of neural network has made great progress [7-10]. Neural network synchronization research is one of the most important research directions of the neural network. Until now, various synchronization control schemes have been proposed, such as impulsive control [11, 12], adaptive control $[13,14]$, sliding mode control $[15,16]$, switching control [17-19], and pinning control [20].

Compared with continuous control, the structure of impulsive control is simpler. The small intermittent control input can achieve the expected control performance [21-24]. The key idea of impulse control is to convert the state of a continuous dynamic system into discontinuous forms through a discrete control input. Since impulsive control allows the system to admit discrete inputs and effectively save network bandwidth resources, it has been studied by many scholars. Liu et al. [25] realized the uniform synchronization of chaotic dynamics system by designing three levels of event-triggered impulsive control. He et al. [26] realized the secure synchronization of multiagent systems under attacks through impulsive control. Qian et al. [27] realized the synchronization of multiagent systems through impulsive control. What is more, there always exists time delay in most practical systems, which should not be ignored in the study of neural network synchronization. For the time delay coupled neural network, the synchronization problem was first discussed in reference [28]. Xu et al. [29] realized the time delay synchronization of the chaotic neural networks based on impulsive control. Xie et al. [30] investigated the synchronization of time-varying delays coupled reaction-diffusion neural networks with pinning impulsive control. Wei et al. [31] realized synchronization of the coupled reaction-diffusion neural networks with timevarying delay by impulsive control. Li et al. [32] investigated the master-slave exponential synchronization of the neural networks with time-varying delays via discontinuous impulsive control. Until now, there are many control methods to realize the synchronization of neural networks. Among them, the research on time-delay impulsive control mainly concentrated on a single neural network system, while the research on the synchronization of coupled neural network with time-delay impulsive control is relatively rare. 
In this paper, the problem of synchronization of delay coupled neural networks under distributed delay impulsive control is studied. Firstly, a time delay coupled neural networks model with $N$ identical nodes is constructed. Secondly, based on the impulsive control method, a distributed delay impulsive controller is designed to achieve synchronization between the drive and response system. Thirdly, through the designed impulsive controller and impulsive delayed inequality, the stability of the synchronization system is analyzed. Finally, numerical examples are given to illustrate the effectiveness of the developed method.
The rest of this paper is organized as follows. In Section 2 , synchronization problem of the time delay coupled neural networks and some premises are proposed. Section 3 presents the main results with proof. A simulation example is provided in Section 4 to illustrate the main results. The summary of this paper is given in Section 5 .

\section{Problem Statement}

Considering a neural networks array consisting of $N$ identical nodes, in which the dynamics of the $i$-th $(1<i<N)$ node is described by the following neural networks:

$$
\begin{cases}\dot{x}_{i}(t)=-C x_{i}(t)+A f\left(x_{i}(t)\right)+B f\left(x_{i}(t-\tau)\right)+\vartheta \sum_{j=1}^{n} d_{i j} \Gamma x_{j}(t)+J, & t \geq t_{0}, \\ x_{i}(s)=\varphi_{i}(s), & s \in\left[t_{0}-\tau, t_{0}\right]\end{cases}
$$

where $x_{i}(t)=\left[x_{i 1}(t), x_{i 2}(t), \ldots, x_{i n}(t)\right]^{T} \in \mathbf{R}^{n}$ denotes the neuron state vector of the $i$-th dynamical node, $i=1,2,3, \ldots, N ; C=\operatorname{diag}\left(c_{1}, \ldots, c_{n}\right) \in \mathbf{R}^{n \times n}$ denotes the positive definite diagonal matrix; $A \in \mathbf{R}^{n \times n}, B \in \mathbf{R}^{n \times n}$ represent the connection weight matrix and the delayed connection weight matrix, respectively; $f\left(x_{i}(t)\right)=$ $\left[f_{1}\left(x_{i 1}(t)\right), f_{2}\left(x_{i 2}(t)\right), \ldots, f_{n}\left(x_{i n}(t)\right)\right]^{T} \in \mathbf{R}^{n} \quad$ represents the neuron activation function satisfying $\left|f_{j}\left(x_{1}\right)-f_{j}\left(x_{2}\right)\right| \leq l_{j}\left|x_{1}-x_{2}\right| \quad$ for all $x_{1}, \quad x_{2} \in \mathbf{R}$, $j=1,2, \ldots, n$, where $l_{j}>0$ are Lipschitz constants; $D=\left(d_{i j}\right)_{N \times N} \in \mathbf{R}^{N \times N}$ is the constant coupling matrix; $\Gamma$ is the inner coupling matrix; $\vartheta>0$ represents coupling strength; $\varphi_{i}(s)$ is the initial condition; $\tau$ denotes the time delay occurring in transmission process; $J$ is an external input.

Let (1) be the drive system and the response system is constructed as follows:

$$
\begin{cases}\dot{y}_{i}(t)=-C y_{i}(t)+A f\left(y_{i}(t)\right)+B f\left(y_{i}(t-\tau)\right)+\vartheta \sum_{j=1}^{n} d_{i j} \Gamma y_{j}(t)+u_{i}(t)+J, & t \geq t_{0}, \\ y_{i}(s)=\psi_{i}(s), & s \in\left[t_{0}-\tau, t_{0}\right],\end{cases}
$$

where $y_{i}(t)=\left[y_{i 1}(t), y_{i 2}(t), \ldots, y_{i n}(t)\right]^{T} \in \mathbf{R}^{n}$ denotes the neuron state vector of the $\mathrm{i}$-th dynamical node, $i=1,2,3, \ldots, N ; u_{i}(t)$ is the impulsive control input; $\psi_{i}(s)$ is the initial condition.

Then, we will design a delayed impulse input to synchronize drive system (1) and response system (2). The impulsive controller is designed by

$$
u_{i}(t)=\sum_{n=1}^{\infty}\left(K \int_{t-r_{n}}^{t} e_{i}(s) \mathrm{d} s\right) \delta\left(t-t_{n}\right)
$$

where $e(t)=y(t)-x(t) ; K \in \mathbf{R}^{n \times n}$ is a gain matrix to be designed; $r_{n}$ denote the distributed time delays in impulse input; $\delta\left(t-t_{n}\right)$ is the Delta function.

When $t \neq t_{n}$ and the controller $u_{i}(t)=0$, the error derivative is

$$
\dot{e}_{i}(t)=-C e_{i}(t)+A g\left(e_{i}(t)\right)+B g\left(e_{i}(t-\tau)\right)+\vartheta \sum_{j=1}^{n} d_{i j} \Gamma e_{j}(t) \text {. }
$$

When $t=t_{n}$, the error derivative is

$$
\begin{aligned}
\dot{e}_{i}(t)= & -C e_{i}(t)+A g\left(e_{i}(t)\right)+B g\left(e_{i}(t-\tau)\right) \\
& +\vartheta \sum_{j=1}^{n} d_{i j} \Gamma e_{j}(t)+K \int_{t-r_{n}}^{t} e_{i}(s) \mathrm{d} s,
\end{aligned}
$$

where $g\left(e_{i}(\cdot)\right)=f\left(e_{i}(\cdot)-x_{i}(\cdot)\right)-f\left(x_{i}(\cdot)\right)$. The above dynamical networks can be rewritten as the following Kronecker product form: 


$$
\left\{\begin{array}{lr}
\dot{e}(t)=-\left(I_{N} \otimes C\right) e(t)+\left(I_{N} \otimes A\right) g(e(t))+\left(I_{N} \otimes B\right) g(e(t-\tau))+\vartheta(D \otimes \Gamma) e(t), & t \neq t_{n} \\
\dot{e}(t)=-\left(I_{N} \otimes C\right) e(t)+\left(I_{N} \otimes A\right) g(e(t))+\left(I_{N} \otimes B\right) g(e(t-\tau))+\vartheta(D \otimes \Gamma) e(t)+\left(I_{N} \otimes K\right) \int_{t-r_{n}}^{t} e(s) \mathrm{d} s, & t=t_{n}
\end{array}\right.
$$

where $\otimes$ denotes the Kronecker product.

Definition 1 (see [33]). The exponential convergence criterion of the distributed delayed impulsive inequality is given:

$$
e(t) \leq \mu_{n} \int_{t-r_{n}}^{t} e(s) \mathrm{d} s, \quad n \in Z_{+},
$$

where $s \in[t-\tau, t], \mu_{n}>0, n \in Z_{+}$are constants. Distributed delays $r_{n}$ satisfy $0<r_{n}<r$, where $r$ is a real constant.

Lemma 1 (see [34]). For the same dimension matrices $H, M$ and the constant $a>0$, then

$$
2 H^{T} M \leq a^{-1} H^{T} H+a M^{T} M .
$$

Assumption 1. There exist positive constants $\zeta>0, n \times n$ matrix $P>0$, and $n \times n$ diagonal matrices $W_{1}>0, W_{2}>0$, the following conditions hold:

$$
\begin{gathered}
\left(\begin{array}{ccc}
\Upsilon & I_{N} \otimes P A & I_{N} \otimes P B \\
* & -W_{1} \otimes I_{N} & 0 \\
* & * & -W_{2} \otimes I_{N}
\end{array}\right)<0, \\
\left(\begin{array}{cc}
\frac{1}{\zeta} I_{N} & P K \\
* & -P
\end{array}\right)<0,
\end{gathered}
$$

where $L=\operatorname{diag}\left(l_{1}, l_{2}, \ldots, l_{n}\right), \quad \Upsilon=-I_{N} \otimes P C+(1 / 2) W_{1} \otimes$ $L^{T} L+(1 / 2) W_{2} \otimes L^{T} L+\vartheta D \otimes P \Gamma$.

\section{Synchronization Analysis}

In this part, we use the Lyapunov stability method and impulsive delayed inequality (7) to derive our main results.

Theorem 1. System (2) can achieve the impulsive synchronization with system (1) under delayed impulsive controller (3), if Assumption 1 holds.

Proof 1. Consider the Lyapunov-like function:

$$
V(t)=\frac{1}{2} e^{T}(t)\left(I_{N} \otimes P\right) e(t)+\frac{1}{2} \int_{t-\tau}^{t} e^{-\alpha(t-s)} e^{T}(s)\left(W_{2} \otimes L^{T} L\right) e(s) \mathrm{d} s .
$$

When $t \neq t_{n}$, the derivative of $V(t)$ along the trajectory of system (6) can be calculated as follows:

$$
\begin{aligned}
\dot{V}(t)= & e^{T}(t)\left(I_{N} \otimes P\right)\left[-\left(I_{N} \otimes C\right) e(t)+\left(I_{N} \otimes A\right) g(e(t))\right. \\
& \left.+\left(I_{N} \otimes B\right) g(e(t-\tau))+\vartheta(D \otimes \Gamma) e(t)\right] \\
& -\frac{\alpha}{2} \int_{t-\tau}^{t} e^{-\alpha(t-s)} e^{T}(s)\left(W_{2} \otimes L^{T} L\right) e(s) \mathrm{d} s \\
& +\frac{1}{2} e^{T}(t)\left(W_{2} \otimes L^{T} L\right) e(t)-\frac{1}{2} e^{-\alpha \tau} e^{T}(t-\tau) \\
& \cdot\left(W_{2} \otimes L^{T} L\right) e(t-\tau) .
\end{aligned}
$$

Based on Lemma 1 , the inequality $2 A^{T} B \leq a^{-1} A^{T} A+$ $a B^{T} B, \quad A, B \in R^{n}, a>0$, we can perform the following calculation:

$$
\begin{aligned}
e^{T}(t)\left(I_{N} \otimes P\right)\left(I_{N} \otimes A\right) g(e(t)) & =\sum_{i=1}^{N} e_{i}^{T}(t) P A g\left(e_{i}(t)\right) \\
& \leq \sum_{i=1}^{N} \frac{1}{2} w_{1 i}^{-1} e_{i}^{T}(t) P A A^{T} P^{T} \\
& \leq \sum_{i=1}^{N} \frac{1}{2} w_{1 i}^{-1} e_{i}^{T}(t) P A A^{T} P^{T} \\
e^{T}(t)\left(I_{N} \otimes P\right)\left(I_{N} \otimes A\right) g(e(t)) & \quad(14) \\
+\frac{1}{2} e^{T}(t)\left(W_{1} \otimes L^{T} L\right) e(t) . &
\end{aligned}
$$$$
\leq \sum_{i=1}^{N} \frac{1}{2} w_{1 i}^{-1} e_{i}^{T}(t) P A A^{T} P^{T} e_{i}(t)+\sum_{i=1}^{N} \frac{1}{2} w_{1 i} g^{T}\left(e_{i}(t)\right) g\left(e_{i}(t)\right)
$$$$
\leq \sum_{i=1}^{N} \frac{1}{2} w_{1 i}^{-1} e_{i}^{T}(t) P A A^{T} P^{T} e_{i}(t)+\sum_{i=1}^{N} \frac{1}{2} w_{1 i} e_{i}^{T}(t) L^{T} L e_{i}(t) .
$$

Changing formula (13) to Kronecker product, we can get

$$
e^{T}(t)\left(I_{N} \otimes P\right)\left(I_{N} \otimes B\right) g(e(t-\tau))
$$$$
\leq \frac{1}{2} e^{T}(t)\left(W_{2}^{-1} \otimes\left(P B B^{T} P^{T}\right)\right) e(t)+\frac{1}{2} e^{T}(t-\tau)\left(W_{2} \otimes L^{T} L\right) e(t-\tau) .
$$

From formulas (14) and (15), formula (9) can be changed to

Similarly, it gives that 


$$
\begin{aligned}
\dot{V}(t)= & e^{T}(t)\left(I_{N} \otimes P\right)\left[-\left(I_{N} \otimes C\right) e(t)+\left(I_{N} \otimes A\right) g(e(t))+\left(I_{N} \otimes B\right) g(e(t-\tau))+\vartheta(D \otimes \Gamma) e(t)\right] \\
& -\frac{\alpha}{2} \int_{t-\tau}^{t} e^{-\alpha(t-s)} e^{T}(s)\left(W_{2} \otimes L^{T} L\right) e(s) \mathrm{d} s+\frac{1}{2} e^{T}(t)\left(W_{2} \otimes L^{T} L\right) e(t)-\frac{1}{2} e^{-\alpha \tau} e^{T}(t-\tau)\left(W_{2} \otimes L^{T} L\right) e(t-\tau) \\
\leq & e^{T}(t)\left[-I_{N} \otimes P C+\frac{1}{2}\left(W_{1}^{-1} \otimes\left(P A A^{T} P^{T}\right)+W_{1} \otimes L^{T} L+W_{2}^{-1} \otimes\left(P B B^{T} P^{T}\right)+W_{2} \otimes L^{T} L\right)+\vartheta(D \otimes P \Gamma)\right] e(t) \\
& +\frac{1}{2}\left(1-e^{-\alpha \tau}\right) e^{T}(t-\tau)\left(W_{2} \otimes L^{T} L\right) e(t-\tau)-\frac{\alpha}{2} \int_{t-\tau}^{t} e^{-\alpha(t-s)} e^{T}(s)\left(W_{2} \otimes L^{T} L\right) e(s) \mathrm{d} s,
\end{aligned}
$$

where $e^{-\alpha \tau}<1$; the following conditions are established:

$$
\dot{V}(t)<0 \text {. }
$$

When $t=t_{n}$, the derivative of $V(t)$ along the trajectory of system (6) can be calculated as follows:

$$
\begin{aligned}
\dot{V}(t)= & e^{T}(t)\left(I_{N} \otimes P\right)\left[-\left(I_{N} \otimes C\right) e(t)+\left(I_{N} \otimes A\right) g(e(t))+\left(I_{N} \otimes B\right) g(e(t-\tau))+\vartheta(D \otimes \Gamma) e(t)+\left(I_{N} \otimes K\right) \int_{t-r_{n}}^{t} e(s) \mathrm{d} s\right] \\
& -\frac{\alpha}{2} \int_{t-\tau}^{t} e^{-\alpha(t-s)} e^{T}(s)\left(W_{2} \otimes L^{T} L\right) e(s) \mathrm{d} s+\frac{1}{2} e^{T}(t)\left(W_{2} \otimes L^{T} L\right) e(t)-\frac{1}{2} e^{-\alpha \tau} e^{T}(t-\tau)\left(W_{2} \otimes L^{T} L\right) e(t-\tau) .
\end{aligned}
$$

From (14) and (15), we have

$$
\begin{aligned}
\dot{V}(t) \leq & e^{T}(t)\left[-I_{N} \otimes P C+\frac{1}{2}\left(W_{1}^{-1} \otimes\left(P A A^{T} P^{T}\right)+W_{1} \otimes L^{T} L+W_{2}^{-1} \otimes\left(P B B^{T} P^{T}\right)+W_{2} \otimes L^{T} L\right)+\vartheta(D \otimes P \Gamma)\right] e(t) \\
& +\frac{1}{2}\left(1-e^{-\alpha \tau}\right) e^{T}(t-\tau)\left(W_{2} \otimes L^{T} L\right) e(t-\tau)+e^{T}(t)\left(I_{N} \otimes P\right)\left(I_{N} \otimes K\right) \int_{t-r_{n}}^{t} e(s) \mathrm{d} s .
\end{aligned}
$$

Based on Lemma 1, we can perform the following calculation:

$$
\begin{aligned}
e^{T}( & \left(I_{N} \otimes P\right)\left(I_{N} \otimes K\right) \int_{t-r_{n}}^{t} e(s) \mathrm{d} s \\
& =e^{T}(t)\left(I_{N} \otimes P K\right) \int_{t-r_{n}}^{t} e(s) \mathrm{d} s \\
& =\sum_{i=1}^{N} e_{i}^{T}(t) P K \int_{t-r_{n}}^{t} e_{i}(s) \mathrm{d} s \\
& \leq \frac{1}{2} \sum_{i=1}^{N}\left(e_{i}^{T}(t) e_{i}(t)+\left(\int_{t-r_{n}}^{t} e_{i}(s) \mathrm{d} s\right)^{T} K^{T} P^{T} P K \int_{t-r_{n}}^{t} e_{i}(s) \mathrm{d} s\right) .
\end{aligned}
$$

It follows from (10) that there exists an inequality such that $-(1 / \zeta) P-P K(P K)^{T}<0$, which together with $(20)$ implies that

$$
\begin{aligned}
& e^{T}(t)\left(I_{N} \otimes P\right)\left(I_{N} \otimes K\right) \int_{t-r_{n}}^{t} e(s) \mathrm{d} s \\
& \quad \leq \frac{1}{2} \sum_{i=1}^{N}\left(e_{i}^{T}(t) P e_{i}(t)-\left(\frac{1}{\zeta} \int_{t-r_{n}}^{t} e_{i}^{T}(s) P e_{i}(s) \mathrm{d} s\right)\right) \\
& \quad \leq \frac{1}{2}\left(e^{T}\left(I_{N} \otimes P\right) e-\frac{1}{\zeta} \int_{t-r_{n}}^{t} e^{T}(s)\left(I_{N} \otimes P\right) e(s) \mathrm{d} s\right) \\
& \quad \leq \frac{1}{2}\left(V(t)-\frac{1}{\zeta} \int_{t-r_{n}}^{t} V(s) \mathrm{d} s\right),
\end{aligned}
$$




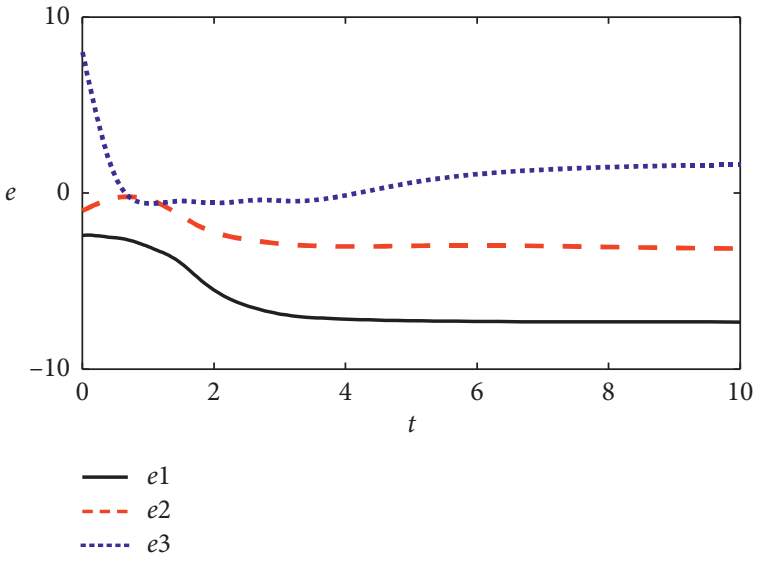

(a)

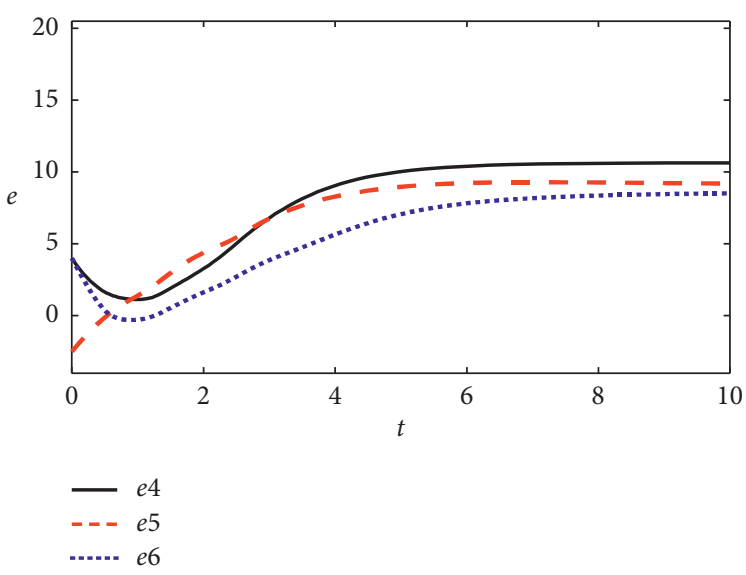

(b)

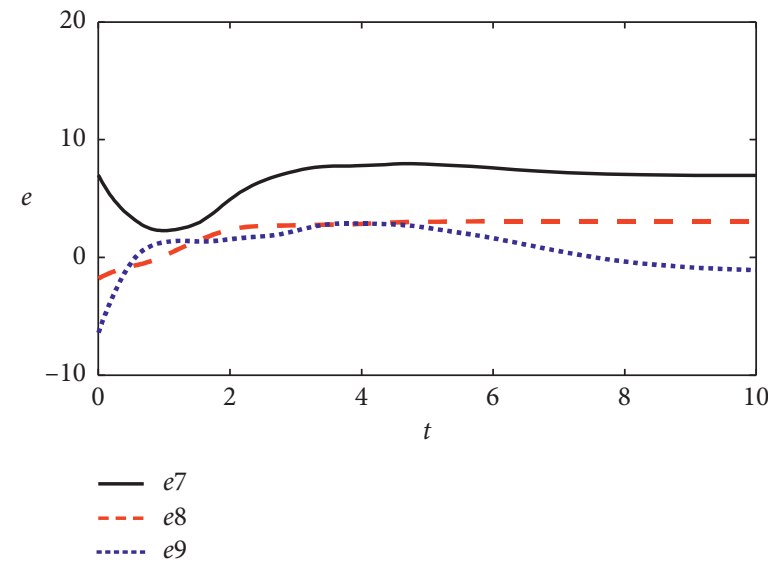

(c)

FIgURE 1: The error trajectories without impulsive control.

where $\zeta=\left(1 / \mu_{n}^{2}\right)$. Then, it can be deduced that $V(t) \leq \mu_{n}^{2} \int_{t-r_{n}}^{t} V(s) \mathrm{d} s$. According to formulas (9) and (21), we can derive that

$$
\dot{V}(t)<0 \text {. }
$$

According to the Lyapunov stability theory, we can obtain $e_{i}(t) \longrightarrow 0$ as $t \longrightarrow \infty$, which means that system (2) can achieve the impulsive synchronization with system (1) under impulsive controller (3). This completes the proof.

\section{Numerical Simulation}

In the simulation, we studied the synchronization problem of the coupled neural network with three nodes, namely, $N=3$. The activation functions are chosen as $f\left(x_{i}\right)=\tanh \left(x_{i}\right)$.

Consider the following neural networks:

$$
\begin{cases}\dot{x}_{i}(t)=-C x_{i}(t)+A f\left(x_{i}(t)\right)+B f\left(x_{i}(t-\tau)\right)+\vartheta_{1} \sum_{j=1}^{n} d_{i j} \Gamma x_{j}(t), & t \geq t_{0}, \\ x_{i}(s)=\varphi_{i}(s), & s \in\left[t_{0}-\tau, t_{0}\right] .\end{cases}
$$




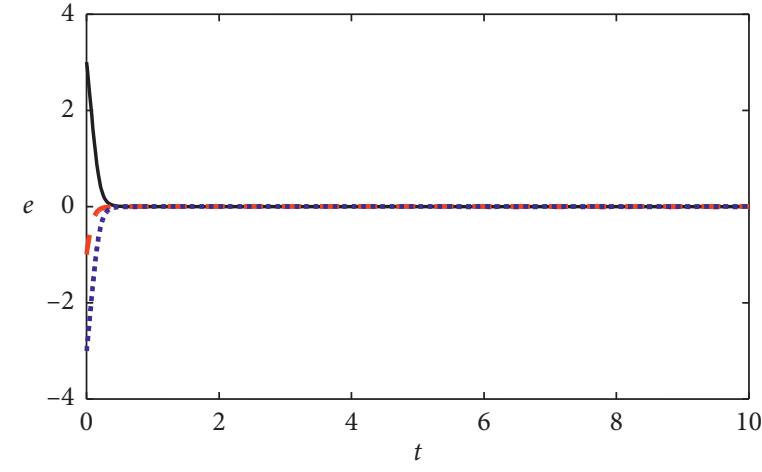

$\begin{array}{ll}- & e 1 \\ --- & e 2 \\ \ldots . . & e 3\end{array}$

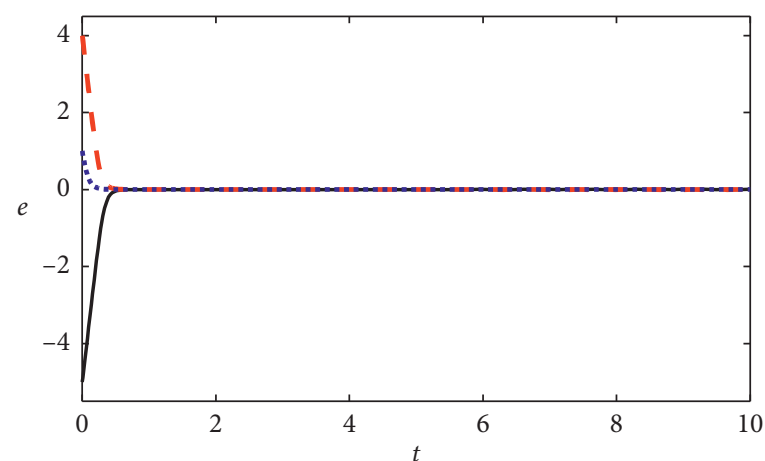

$-e 4$

...... e6

(a)

(b)

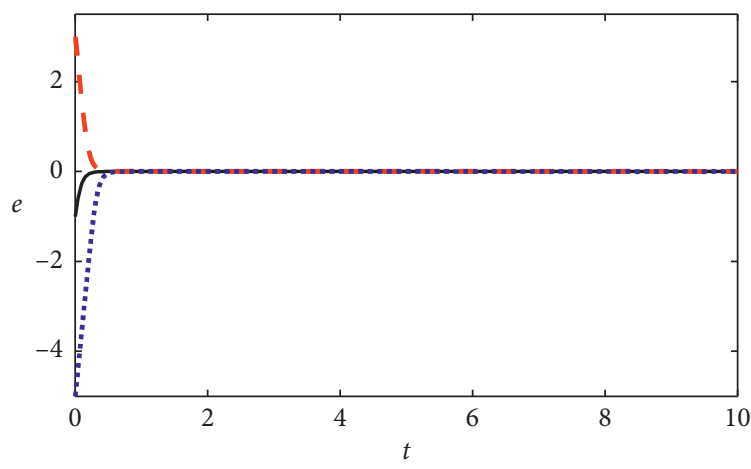

$\begin{array}{ll}- & e 7 \\ --- & e 8 \\ \cdots & e 9\end{array}$

(c)

Figure 2: The error trajectories with impulsive control.

Consider the following response system:

$$
\begin{cases}\dot{y}_{i}(t)=-C y_{i}(t)+A f\left(y_{i}(t)\right)+B f\left(y_{i}(t-\tau)\right)+\vartheta_{2} \sum_{j=1}^{n} d_{i j} \Gamma y_{j}(t)+u_{i}(t), & t \geq t_{0}, \\ y_{i}(s)=\psi_{i}(s), & s \in\left[t_{0}-\tau, t_{0}\right],\end{cases}
$$

where $\tau=1, i=1,2,3, j=1,2,3, \vartheta_{1}=0.5, \vartheta_{2}=5$, initial condition $\varphi_{1}(s)=[0.5,0.6,0.7]^{T}, \varphi_{2}(s)=[0.6,0.7,0.8]^{T}$, $\varphi_{3}(s)=[0.7,1,1.5]^{T}, \quad \psi_{1}(s)=[1,1.5,2]^{T}, \quad \psi_{2}(s)=[1.4$,
1.6, 1.5 $]^{T}, \quad \psi_{3}(s)=\left[\begin{array}{lll}1.6, & 1.7, \quad 1.8\end{array}\right]^{T}, \quad$ and $\quad s \in$ $[-1,0]$, and the parameter matrices $C, A, B, D$, and $\Gamma$ are given by 


$$
\begin{aligned}
C_{1} & =\left[\begin{array}{lll}
1 & 0 & 0 \\
0 & 1 & 0 \\
0 & 0 & 1
\end{array}\right], \\
C_{2} & =\left[\begin{array}{lll}
2 & 0 & 0 \\
0 & 1 & 0 \\
0 & 0 & 1
\end{array}\right], \\
A_{1} & =\left[\begin{array}{ccc}
1 & 3 & 2 \\
-0.1 & 2 & 0.5 \\
3 & 1 & -3
\end{array}\right], \\
A_{2} & =\left[\begin{array}{ccc}
1 & 0.6 & -1 \\
-1 & 2 & 0.1 \\
3 & 0.5 & -3
\end{array}\right], \\
B_{1} & =\left[\begin{array}{ccc}
0.2 & 1 & 0.5 \\
3 & 0.4 & 0.1 \\
0.4 & 0.6 & 2
\end{array}\right], \\
D_{1} & =D_{2}=\left[\begin{array}{ccc}
1 & 0.3 & 2 \\
0.5 & 1 & 0.6 \\
3 & 1 & 0.4
\end{array}\right],
\end{aligned}
$$

When there is no control input (i.e., $u(t)=0$ ), system (24) cannot be synchronized with system (23), see Figure 1. Next, we consider distributed delayed impulsive control to achieve the synchronization between system (23) and system (24). The control input $u(t)$ is given by (3) with $r_{n}=1.2$. Choose $\zeta=0.05$, using the MATLAB LMI toolbox, and the following feasible solutions can be derived:

$$
\begin{aligned}
P & =\left[\begin{array}{ccc}
0.7025 & -0.4528 & -0.0353 \\
-0.4528 & 0.8421 & -0.0435 \\
-0.0353 & -0.0435 & 0.2865
\end{array}\right], \\
W_{1} & =\left[\begin{array}{ccc}
0.3272 & 0 & 0 \\
0 & 0.4158 & 0 \\
0 & 0 & 0.4191
\end{array}\right], \\
W_{2} & =\left[\begin{array}{ccc}
0.4930 & 0 & 0 \\
0 & 0.3724 & 0 \\
0 & 0 & 0.3405
\end{array}\right] .
\end{aligned}
$$

Thus, the gain matrix $K$ is derived as follows:

$$
K=\left[\begin{array}{ccc}
0.3756 & -0.1417 & 0.0184 \\
-0.1417 & -0.3321 & -0.0200 \\
0.0184 & -0.0200 & 0.5108
\end{array}\right] \text {. }
$$

For simulation, if we take $t_{n}=0.07 n, n \in Z$, then the synchronization error is shown in Figure 2.

\section{Conclusion}

In this paper, the synchronization of coupled neural networks is studied by distributed delayed impulsive control. Based on the impulsive control theory and Lyapunov stability theory, a distributed delayed impulsive controller is proposed. The proposed inequality fully considers the synchronization of the response system and the drive system under different time conditions. The numerical simulation proves the feasibility and effectiveness of our proposed scheme. In the future, we will deal with finite time synchronization of coupled neural networks through distributed delayed impulsive control.

\section{Data Availability}

The data used to support the findings of this study are available from the corresponding author upon request.

\section{Conflicts of Interest}

The authors declare that they have no conflicts of interest.

\section{Acknowledgments}

The work was supported by the National Natural Science Foundation of China (Grant no. 61775198), Science and Technology Project of Henan Province (Grant nos. 192102210083 and 202102210317), Key Scientific Research Projects of Universities in Henan Province (Grant no. 20A413012), and Science and Technology Innovation Team Project of Henan Province (Grant no. 19IRTSTHN013).

\section{References}

[1] S. Arik, "An analysis of exponential stability of delayed neural networks with time varying delays," Neural Networks, vol. 17, no. 7, pp. 1027-1031, 2004.

[2] X. Ye, J. Mou, C. Luo, and Z. Wang, "Dynamics analysis of Wien-bridge hyperchaotic memristive circuit system," Nonlinear Dynamics, vol. 92, no. 3, pp. 923-933, 2018.

[3] L. O. Chua and L. Yang, "Cellular neural networks: applications," IEEE Transactions on Circuits and Systems, vol. 35, no. 10, pp. 1273-1290, 1998.

[4] J. W. Sun, G. Y. Han, Z. G. Zeng, and Y. F. Wang, "Memristorbased neural network circuit of full-function pavlov associative memory with time delay and variable learning rate," IEEE Transactions on Cybernetics, vol. 10, p. 1109, 2019.

[5] X. Liu, X. Shen, Y. Zhang, and Q. Wang, "Stability criteria for impulsive systems with time delay and unstable system 
matrices," IEEE Transactions on Circuits and Systems I: Regular Papers, vol. 54, no. 10, pp. 2288-2298, 2007.

[6] H. Su, Y. Sun, and Z. Zeng, "Semiglobal observer-based nonnegative edge consensus of networked systems with actuator saturation," IEEE Transactions on Cybernetics, vol. 50, no. 6 , pp. 2827-2836, 2020.

[7] U. Fory and M. Bodnar, "Time delays in proliferation process for solid avascular tumour," Math and Computer Modelling, vol. 37, no. 11, pp. 1201-1209, 2003.

[8] W. Yu, J. Cao, and G. Chen, "Stability and Hopf bifurcation of a general delayed recurrent neural network," IEEE Transactions on Neural Networks, vol. 19, no. 5, pp. 845-854, 2008.

[9] H. Su, J. Zhang, and Z. Zeng, "Formation-containment control of multi-robot systems under a stochastic sampling mechanism," Science China Technological Sciences, vol. 63, no. 6, pp. 1025-1034, 2020.

[10] F. F. Yang, J. Mou, J. Liu, C. G. Ma, and H. Z. Yan, "Characteristic analysis of the fractional-order hyperchaotic complex system and its image encryption application," Signal Processing, vol. 169, 2020.

[11] Q. Zhang, J. Lu, and J. Zhao, "Impulsive synchronization of general continuous and discrete-time complex dynamical networks," Communications in Nonlinear Science and $\mathrm{Nu}$ merical Simulation, vol. 15, no. 10, pp. 1063-1070, 2010.

[12] J. Zhou, L. Xiang, and Z. Liu, "Synchronization in complex delayed dynamical networks via impulsive control," Physica A: Statistical Mechanics and Its Applications, vol. 384, no. 2, pp. 684-692, 2007.

[13] W. Xu, S. Zhu, X. Fang, and W. Wang, "Adaptive synchronization of memristor-based complex-valued neural networks with time delays," Neurocomputing, vol. 364, no. 28, pp. 119-128, 2019.

[14] Q. Wang and J. L. Wu, "Finite-time output synchronization of undirected and directed coupled neural networks with output coupling," IEEE Transactions on Neural Neworks and Learning Systems, vol. 10, p. 1109, 2020.

[15] M. P. Aghababa, K. Sohrab, and G. Alizadeh, "Finite-time synchronization of two different chaotic systems with unknown parameters via sliding mode technique," Applied Mathematical Modelling, vol. 35, no. 10, pp. 3080-3091, 2011.

[16] J. Sun, Y. Wu, G. Cui, and Y. Wang, "Finite-time real combination synchronization of three complex-variable chaotic systems with unknown parameters via sliding mode control," Nonlinear Dynamics, vol. 88, no. 3, pp. 1677-1690, 2017.

[17] P. Selvaraj, R. Sakthivel, and O. M. Kwon, "Finite-time synchronization of stochastic coupled neural networks subject to Markovian switching and input saturation," Neural Networks, vol. 105, pp. 154-165, 2018.

[18] D. Zhang, J. Cheng, J. Cao, and D. Zhang, "Finite-time synchronization control for semi-Markov jump neural networks with mode-dependent stochastic parametric uncertainties," Applied Mathematics and Computation, vol. 344345, no. 1, pp. 230-242, 2019.

[19] Y. Wu, J. Cao, Q. Li, A. Alsaedi, and F. E. Alsaadi, "Finite-time synchronization of uncertain coupled switched neural networks under asynchronous switching," Neural Networks, vol. 85, pp. 128-139, 2017.

[20] Q. Song, J. Cao, and F. Liu, "Pinning synchronization of linearly coupled delayed neural networks," Mathematics and Computers in Simulation, vol. 86, pp. 39-51, 2012.

[21] W.-H. Chen and W. X. Zheng, "Exponential stability of nonlinear time-delay systems with delayed impulse effects," Automatica, vol. 47, no. 5, pp. 1075-1083, 2011.
[22] Z.-H. Guan, G.-S. Han, J. Li, D.-X. He, and G. Feng, "Impulsive multiconsensus of second-order multiagent networks using sampled position data," IEEE Transactions on Neural Networks and Learning Systems, vol. 26, no. 11, pp. 26782688, 2015.

[23] Z. Huang, J. Cao, J. Li, and H. Bin, "Quasi-synchronization of neural networks with parameter mismatches and delayed impulsive controller on time scales," Nonlinear Analysis: Hybrid Systems, vol. 33, pp. 104-115, 2019.

[24] X. Yang, X. Li, X. Li, Q. Xi, and P. Duan, "Review of stability and stabilization for impulsive delayed systems," Mathematical Biosciences \& Engineering, vol. 15, no. 6, pp. 14951515, 2018.

[25] B. Liu, Z. Sun, Y. Luo, and Y. Zhong, "Uniform synchronization for chaotic dynamical systems via event-triggered impulsive control," Physica A: Statistical Mechanics and Its Applications, vol. 531, no. 1, p. 121725, 2019.

[26] W. He, X. Gao, W. Zhong, and F. Qian, "Secure impulsive synchronization control of multi-agent systems under deception attacks," Information Sciences, vol. 459, pp. 354-368, 2018.

[27] T. Qian, T. Yu, and B. Cui, "Adaptive synchronization of multi-agent systems via variable impulsive control," Journal of the Franklin Institute, vol. 355, no. 15, pp. 7490-7508, 2018.

[28] W. Lu and T. Chen, "Synchronization of coupled connected neural networks with delays," IEEE Transactions on Circuits and Systems I: Regular Papers, vol. 51, no. 12, pp. 2491-2503, 2004.

[29] Z. Xu, D. Peng, and X. Li, "Synchronization of chaotic neural networks with time delay via distributed delayed impulsive control," Neural Networks, vol. 118, pp. 332-337, 2019.

[30] X. Xie, X. Liu, H. Xu, X. Luo, and G. Liu, "Synchronization of coupled reaction-diffusion neural networks: delay-dependent pinning impulsive control," Communications in Nonlinear Science and Numerical Simulation, vol. 79, Article ID 104905, 2019.

[31] P.-C. Wei, J.-L. Wang, Y.-L. Huang, B.-B. Xu, and S.-Y. Ren, "Impulsive control for the synchronization of coupled neural networks with reaction-diffusion terms," Neurocomputing, vol. 207, no. 26, pp. 539-547, 2016.

[32] X. Li, J.-a. Fang, and H. Li, "Master-slave exponential synchronization of delayed complex-valued memristor-based neural networks via impulsive control," Neural Networks, vol. 93, pp. 165-175, 2017.

[33] Y. Kan, J. Lu, J. Qiu, and J. Kurths, "Exponential synchronization of time-varying delayed complex-valued neural networks under hybrid impulsive controllers," Neural Networks, vol. 114, pp. 157-163, 2019.

[34] S. Boyd, L. E. Ghaoui, E. Feron, and V. Balakrishnan, Linear Matrix Inequality in Systems and Control Theory, SIAM, Philadelphia, PA, USA, 1994. 